\title{
Prevalence and Impact of Coagulation Dysfunction in COVID-19 in China: A Meta-Analysis
}

\author{
${ }^{1}$ Department of Gastrointestinal and Pancreatic Surgery, Zhejiang \\ Provincial People's Hospital (People's Hospital of Hangzhou Medical \\ College), Key laboratory of Gastroenterology of Zhejiang Province, \\ Hangzhou, Zhejiang, China \\ 2 Department of Molecular and Cell Biology, Department of Statistics, \\ College of Letters and Science, University of California, Berkeley, \\ California, United States \\ ${ }^{3}$ Department of Clinical Medicine, Bengbu Medical College Graduate \\ School, Bengbu, Anhui, China \\ ${ }^{4}$ Department of Critical Care Medicine, Zhejiang Provincial People's \\ Hospital (People's Hospital of Hangzhou Medical College), \\ Hangzhou, Zhejiang, China
}

Shanen Jin ${ }^{1, *}$ Yiyang Jin ${ }^{2, *}$ Bai $\mathrm{Xu}^{3}$ Jun Hong ${ }^{4} \quad$ Xianghong Yang ${ }^{4}$

Address for correspondence Xianghong Yang, PhD, Department of Critical Care Medicine, Zhejiang Provincial People's Hospital (People's Hospital of Hangzhou Medical College), 158 Shangtang Road, Hangzhou, Zhejiang 310014, China (e-mail: jyy623@163.com).

\begin{abstract}
Keywords

- COVID-19

- coagulopathy

- disseminated intravascular coagulation

- venous thromboembolism

- anticoagulation

Background The aim of this meta-analysis is to assess the prevalence of coagulation dysfunction in Chinese COVID-19 patients and to determine the association of coagulopathy with the severity and prognosis of COVID-19.

Methods A meta-analysis of the prevalence of different abnormal coagulation indicators in COVID-19 patients in China was performed. The difference of coagulation indicators and the incidence of DIC were compared between severe cases and nonsevere cases as well as nonsurvivors and survivors, respectively.

Results A total of 22 Chinese studies involving 4,889 confirmed COVID-19 inpatients were included. The average D-dimer value of COVID-19 patients is $0.67 \mu \mathrm{g} / \mathrm{mL}(95 \%$ confidence interval [Cl]: $0.56-0.78)$, and $29.3 \%$ (95\% Cl: $20.1-38.5 \%$ ) of patients showed elevated D-dimer values. Severe patients had significantly higher D-dimer levels and prolonged prothrombin time (PT) compared with nonsevere patients. Nonsurvivors had significantly higher D-dimer levels, prolonged PT, and decreased platelet count compared with survivors. In total, 6.2\% (95\% Cl: 2.6-9.9\%) COVID-19 patients were complicated by disseminated intravascular coagulation (DIC), in which the log risk ratio in nonsurvivors was 3.267 (95\% Cl: 2.191-4.342, Z =5.95, $p<0.05$ ) compared with that in survivors.

Conclusion The prevalence of coagulopathy in Chinese COVID-19 inpatients is high, and both the abnormal coagulation indicators and DIC are closely associated with the severity and poor prognosis of these COVID-19 patients. Therefore, attention should be paid to coagulation dysfunction in COVID-19 patients. Closely monitoring of coagulation indicators and application of appropriate anticoagulation may improve the prognosis of COVID-19 inpatients in China.
\end{abstract}

* These authors contributed equally to this article.

received

May 15, 2020

accepted after revision

June 26, 2020 (c) 2020 Georg Thieme Verlag KG Stuttgart - New York
DOI https://doi.org/

10.1055/s-0040-1714369. ISSN 0340-6245. 


\section{Introduction}

At the end of 2019, a novel coronavirus, named severe acute respiratory syndrome coronavirus 2 (SARS-CoV-2), resulted in an acute respiratory illness epidemic in Wuhan, China., The World Health Organization termed this illness as Coronavirus Disease 2019 (COVID-19). ${ }^{3}$ By May 3, 2020, there are $3,349,786$ laboratory-confirmed cases of COVID-19 globally and the global mortality related to COVID-19 has reached $7.11 \%$ (238,268 deaths). ${ }^{4}$

The clinical features of COVID-19 are similar to the characteristics of SARS, primarily manifested as an acute respiratory illness with interstitial and alveolar pneumonia. ${ }^{5-8}$ Previous literatures have shown that SARS patients were always complicated by coagulation disorders, showing prolonged activated partial thromboplastin time (APTT), thrombocytopenia, elevated D-dimer, and complications with disseminated intravascular coagulation (DIC), and the incidence rates for which were $50-63,40-45,50$, and $2.5 \%$, respectively. ${ }^{9,10}$ Based on our frontline experience in Wuhan, we found that coagulation dysfunction is also common in COVID-19 patients. But different studies have shown that the inconsistent levels of change of coagulation indicators in COVID-19 patients are inconsistent and the incidence of DIC ranges from 0.1 to $8.74 \% .^{11-13}$ To get a more complete understanding of coagulopathy in COVID-19 in China, we here provide a meta-analysis to not only evaluate the prevalence of coagulation dysfunction in COVID-19 patients, but also to assess the risk of coagulation abnormalities in severe patients and nonsurvivors compared with nonsevere patients and survivors, respectively. The results are helpful for the Chinese clinicians to standardize the management of COVID-19 patients complicated with coagulation dysfunction.

\section{Methods}

Data Source, Search Strategy, and Exclusion Criteria

A systematic literature search was performed on PubMed and Embase from December 2019 to April 2020 to identify all studies that relate to coagulation dysfunction caused by COVID-19 infection or provide general clinical features of COVID-19 patients in China. The keywords for the literature search were combinations of "COVID-19," "2019-nCoV," and "novel coronavirus" with "clinical characteristics," "clinical features," "coagulation," "coagulopathy," and "DIC." A total of 406 studies were identified, and the following selection criteria were used to exclude the studies that did not provide useful information: (1) duplicated studies, (2) studies with sample sizes smaller than 10,(3) studies without useful information on clinical characteristics or coagulation indicators, (4) studies that focus only on children or infants, (5) case reports, reviews of editorials, letters, and comments, and (6) studies written in Chinese (to prevent data duplication). Two investigators performed the selection process independently, and a third investigator helped to decide whether the article should be included if the first two investigators were in disagreement. The data selection process is illustrated by a flow chart (-Fig. 1 ), and a total of 22 articles are included in this study. ${ }^{11-32}$

\section{Data Extraction}

We extract the main characteristic of patients (study population, age, sex, mortality, proportion of intensive care unit [ICU] admission, proportion of severe cases, and proportion of patients with underlying diseases) and following parameters from the 22 selected articles: prothrombin time (PT), APTT, normal range of PT and APTT, D-dimer, fibrinogen, platelet (PLT), incidence of DIC, and proportion of patients with prolonged or shortened PT, prolonged or shortened APTT, elevated or decreased PLT, elevated D-dimer, and time of measurement taken (hospital admission or not available). PT and APTT were measured in seconds, D-dimer was measured in $\mu \mathrm{g} / \mathrm{mL}$, fibrinogen was measured in $\mathrm{g} / \mathrm{L}$, PLT count was measured in $\times 10^{9} / \mathrm{L}$, and the rest were proportions. Some of the studies did not provide the mean and standard deviation of coagulation indicator, but provided their median ( $\mathrm{m}$ ) and the lower (q1) and upper quartiles (q3) instead. An estimation is applied to these data to get the approximate mean and standard deviation with the following formula: mean $=(\mathrm{q} 1+\mathrm{m}+\mathrm{q} 3) / 3$, and standard deviation $=(\mathrm{q} 3-\mathrm{q} 1) / 1.35 .{ }^{33}$ We also separate patients into groups of severe and nonsevere patients or groups of survivor and nonsurvivors for further analysis. These data are shown in -Tables 1 and 2.

Underlying disease was defined as various preexisting chronic diseases, which included cardiovascular disease, hypertension, diabetes, chronic heart failure, chronic renal disease, chronic obstructive pulmonary disease, cancer, and so on. The definition was not explicit in some papers and may have varied. DIC was defined as meeting the International Thrombosis and Hemostasis Association (ISTH) criteria, and ISTH score $>5$ is diagnosed as overt-DIC. The severe group was defined as being admitted to ICU or belongs to severe and critical cases according to the data in the studies.

\section{Data Analysis}

The meta-analysis was performed with $\mathrm{R}$ version 3.6.3. Forest plots were made to illustrate the mean and corresponding $95 \%$ confidence intervals (CIs) of different coagulation indicators of COVID-19 patients and the average proportion and 95\% CI of patients with DIC. As the normal ranges of PT and APTT values from different laboratories vary greatly, thus we also calculate the PT and APTT as the ratio to the upper limit of the laboratory-specific normal range so that the data entered into the meta-analysis become more consistent. We also compared the mean coagulation indicators in severe patients with those in nonsevere patients, and in survivors with those in nonsurvivors. The differences of coagulation indicators between the two groups were illustrated with forest plots of mean difference and corresponding $95 \% \mathrm{CI}$ of the indicators between patients in the two groups as all the data are measured in the same units. A forest plot of risk ratio (RR and $95 \% \mathrm{CI}$ ) was also made to analyze the relative risk of nonsurvivors compared with survivors to develop DIC. For the meta-analysis, the heterogeneity level was defined based on the $I^{2}$ index calculated: if $I^{2}<25 \%$, the data are homogenous; if $25 \% \leq I^{2} \leq 50 \%$, there is low heterogeneity within the data; if $50 \% \leq I^{2} \leq 75 \%$, moderate heterogeneity is included; and $I^{2} \geq 75 \%$ represents high heterogeneity. ${ }^{34}$ Different models 


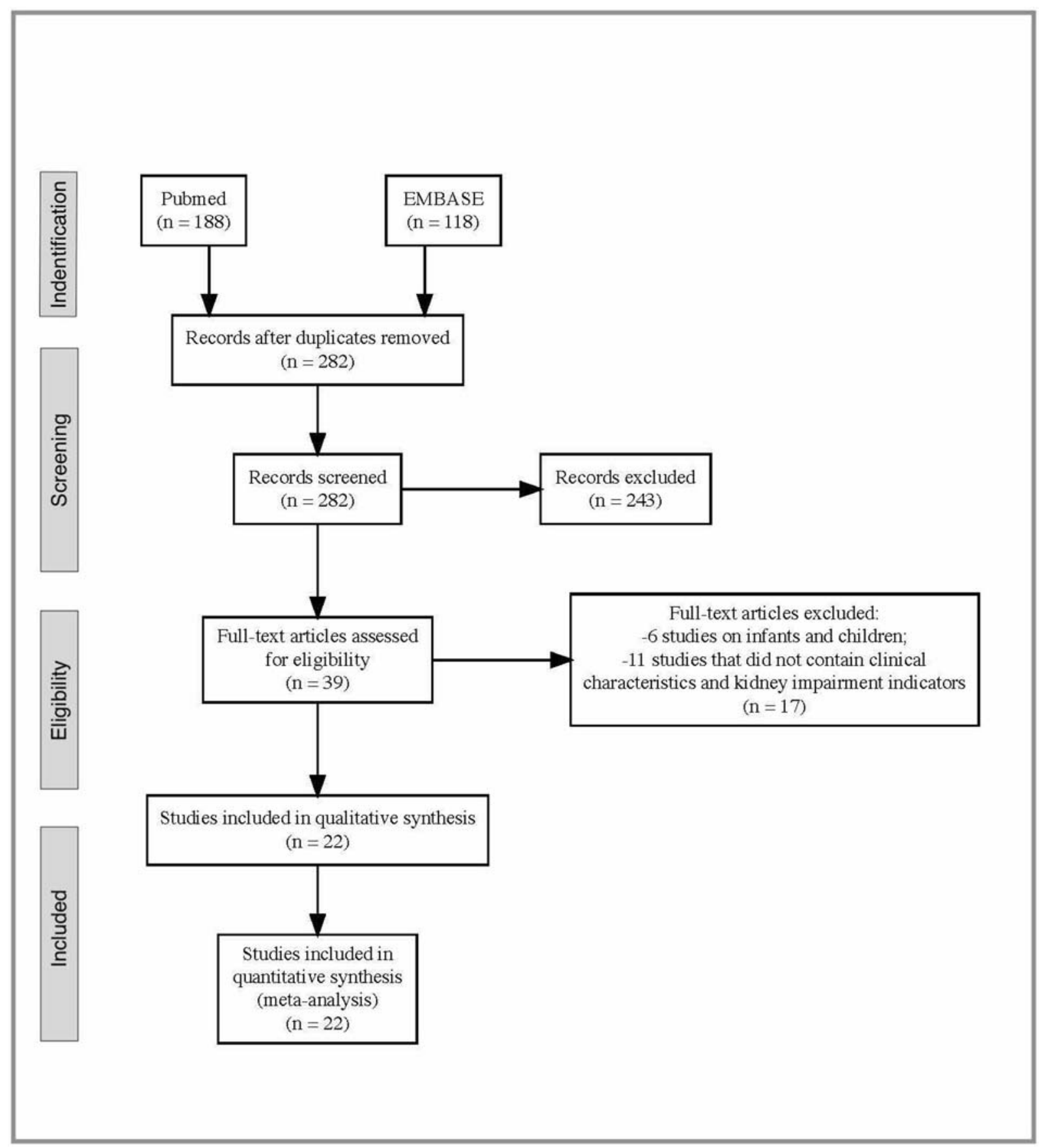

Fig. 1 Flow chart of the process to screen and select the 22 studies included in this meta-analysis.

are used to calculate the weight for each study based on the $I^{2}$ index and heterogeneity: we incorporated a fixed effect model (inverse variance) to pool the data if $I^{2}$ is $\leq 50 \%$, and we used a random effect model (DerSimonian-Laird) to pool the data if $I^{2}>50 \% .{ }^{35}$ We used 0.05 as the threshold for significant tests in this article: a $p$-value $<0.05$ indicated statistical significance.

\section{Results}

\section{Selected Studies and Baseline Characteristics}

The literature search initially identified 247 articles from PubMed and 159 articles from Embase, in which 124 were identified as duplicates and removed. Eventually, 39 studies that might fulfill our selection criteria were selected. For the 39 selected studies, the team reviewed the full text and identified 22 papers that included useful coagulation indicators and clinical features for the meta-analysis. All the 22 papers focused on patients in China from late-December 2019 to late-February 2020 and were published in 2020. The sample size of groups varied from 41 to 1,099 , and a total number of 4,889 patients were included in the 22 studies. The median age was 55.36 years old. The overall proportion of male is $55.44 \%$, the average proportion of COVID-19 patients with underlying disease was $42.6 \%$ (95\% $\mathrm{CI}$ : $35.5-49.6 \%$ ), and the average mortality rate was $11.8 \%$ (95\% CI: 8.7-14.9\%) (- Table 1 and -Supplementary Fig. S1, 


\begin{tabular}{|c|c|c|c|c|c|c|c|c|c|c|c|c|c|c|c|c|c|c|c|}
\hline 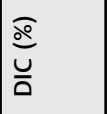 & $\begin{array}{l}\hat{\jmath} \\
\hat{\infty} \\
\infty \\
\underline{0} \\
\underline{0}\end{array}$ & $\frac{s}{z}$ & $\frac{s}{z}$ & $\frac{s}{2}$ & $\frac{\pi}{2}$ & 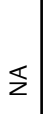 & $\left|\begin{array}{l}\bar{\infty} \\
\bar{v}\end{array}\right|$ & $\Sigma$ & 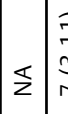 & & $\begin{array}{l}\mathbf{s} \\
\mathbf{2}\end{array}$ & $\frac{s}{z}$ & $\frac{s}{z}$ & $\bar{z}$ & $\frac{s}{z}$ & 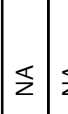 & 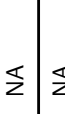 & $\frac{\pi}{z}$ & $\Delta$ \\
\hline 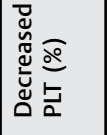 & $\bar{z}$ & 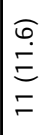 & $\frac{s}{z}$ & $\frac{s}{z}$ & $\Sigma$ & 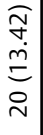 & $\Sigma$ & $\frac{\tilde{z}}{z}$ & $\Sigma$ & $\begin{array}{l}\mathbf{z} \\
\mathbf{z}\end{array}$ & $\Sigma$ & $\begin{array}{l}\Sigma \\
z\end{array}$ & $\Sigma \sum_{n}^{n}$ & $\begin{array}{l}n \\
v\end{array}$ & $\S$ & \begin{tabular}{|l|}
2 \\
0 \\
0 \\
0 \\
0 \\
0 \\
0
\end{tabular} & 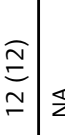 & $\begin{array}{l}z \\
z\end{array}$ & $\frac{4}{2}$ \\
\hline 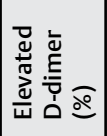 & $\underline{\Sigma}$ & 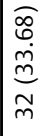 & $\Sigma$ & $\Sigma$ & $\Sigma$ & $\begin{array}{l}\widehat{a} \\
\dot{+} \\
\dot{+} \\
\bar{\sim}\end{array}$ & $\begin{array}{l}\sqrt[\Omega]{n} \\
\stackrel{n}{m} \\
m\end{array}$ & $\Sigma$ & 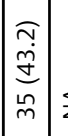 & $\Sigma$ & 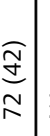 & & $\Sigma \mid \Sigma$ & $\underline{z}$ & $\mid \begin{array}{l}\widehat{0} \\
\dot{0} \\
\tilde{\omega} \\
\sigma \\
\sigma\end{array}$ & $\mid \begin{array}{l}a \\
\dot{0} \\
\dot{d} \\
\tilde{N} \\
\tilde{N}\end{array}$ & 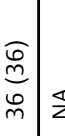 & 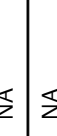 & $\mid \begin{array}{l}z \\
z \\
z \\
z\end{array}$ \\
\hline
\end{tabular}

\begin{tabular}{|c|c|c|c|c|c|c|c|c|c|c|c|c|c|c|c|c|c|c|c|}
\hline 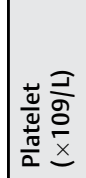 & $\underline{z}$ & $\Sigma$ & 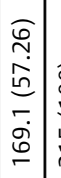 & 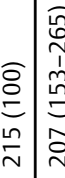 & 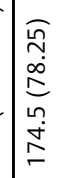 & 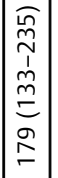 & $\begin{array}{l}\bar{\sigma} \\
\bar{\sigma} \\
\tilde{n} \\
\tilde{D} \\
\tilde{n} \\
\underline{\sigma} \\
\underline{0}\end{array}$ & $\Sigma$ & $\Sigma$ & 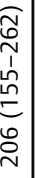 & $\mathbb{z}$ & 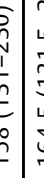 & 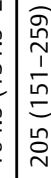 & $\Sigma$ & $\stackrel{\circ}{\circ}$ & 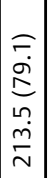 & $\begin{array}{c}\widehat{\infty} \\
\underset{\sim}{0} \\
\tilde{\infty} \\
\tilde{\infty} \\
\infty \\
\dot{m} \\
m\end{array}$ & & $\begin{array}{l}\tilde{E} \\
0 \\
1 \\
1 \\
\tilde{N} \\
\tilde{N} \\
0 \\
0 \\
\end{array}$ \\
\hline
\end{tabular}



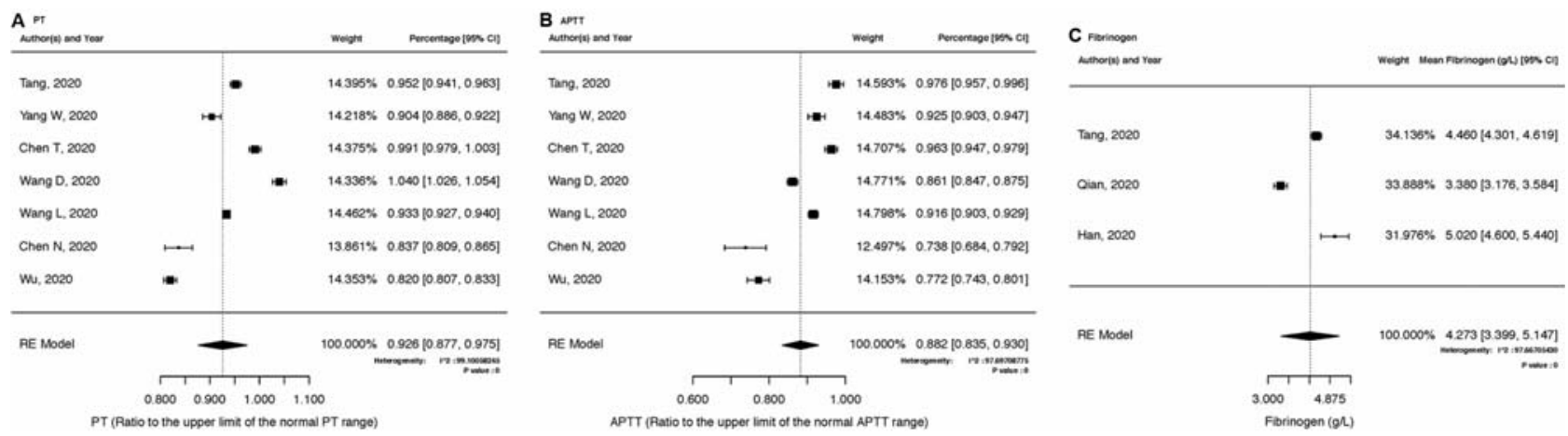

Fig. 2 (A) Forest plot of the average PT of COVID-19 patients measured in the ratio to the upper limit of the laboratory-specific normal range. (B) Forest plot of the average APTT of COVID-19 patients measured in the ratio to the upper limit of the laboratory-specific normal range. (C) Forest plot of average fibrinogen of COVID-19 patients. Heterogeneity is defined based on the $l^{2}$ index calculated, and random effect models are used to pool the database on the heterogeneity. APTT, activated partial thromboplastin time; PT, prothrombin time.

available in the online version). The degree of severity of COVID-19 were defined in accordance with the Diagnosis and Treatment Protocol of COVID-19 released by National Health Commission of China by all of the articles, except the article ${ }^{14}$ which was based on the American Thoracic Society guidelines for community-acquired pneumonia. Most of the studies showed that coagulation indicators were measured on the day of hospital admission, and the data were not available in four articles. ${ }^{12,16,18,20}$

\section{Primary Outcomes}

The meta-analysis showed that the average PT for COVID-19 patients was 12.20 seconds ( $95 \% \mathrm{CI}: 11.52-12.84$ ) and the average APTT was 31.53 seconds $(95 \%$ Cl: $28.46-$ 34.60; - Supplementary Fig. S2, available in the online version). Meanwhile, the average PT and APTT are 92.6\% (95\% CI: $87.7-97.5 \%$ ) and $88.2 \%$ (83.5-93.0\%) of the upper limit of the normal range, respectively. The average fibrinogen level was $4.24 \mathrm{~g} / \mathrm{L}$ (95\% CI: 3.40-5.15; - Fig. 2). The result also showed that the mean PLT count for COVID-19 patients was $186.34 \times 10^{9} / \mathrm{L}$ (95\% CI: 175.84-196.85), and 10.9\% (95\%

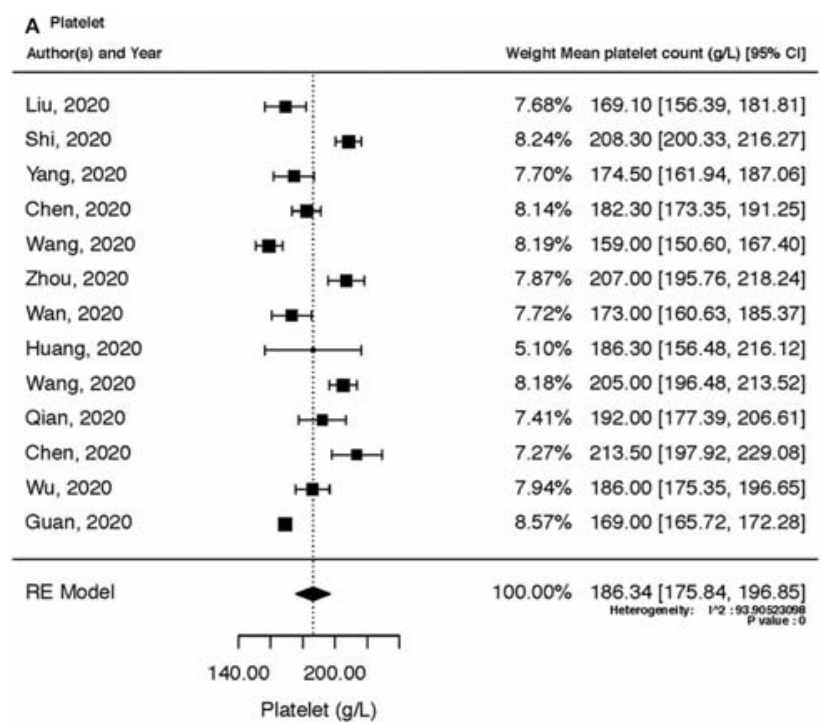

CI: 8.1-13.6\%) of COVID-19 patients on average showed decreased PLT levels ( - Fig. 3 ). In addition, we found that the average D-dimer concentration for COVID-19 patients calculated from the meta-analysis was $0.67 \mu \mathrm{g} / \mathrm{mL}(95 \% \mathrm{CI}$ : $0.56-0.78$ ), and that $29.3 \%$ (95\% CI: 20.1-38.5\%) of COVID-19 patients showed elevated D-dimer values (-Fig. 4). All the above data showed calculated $I^{2}$ in the range of 76.35 to 99.50\%, except for $I^{2}$ index of 4.79 for the data of percentage of patients with decreased PLT. Therefore, the fixed effect model was applied to the decreased PLT level data, whereas the random effect model was used to pool all the other data.

Next, we separated the data into groups of severe and nonsevere COVID-19 patients, and calculated the mean difference of coagulation indicators including PT, APTT, Ddimer value, and PLT count between patients of the two groups. The meta-analysis showed that the average PT for severe patients was 0.65 seconds (95\% CI: 0.36-0.95, $Z=4.35, p<0.05$ ), longer than that of nonsevere patients ( -Fig. 5A). The mean difference of APTT between severe and nonsevere patients was -0.01 second $(95 \% \mathrm{CI}:-2.58-2.56$, $Z=0, p=0.99$ ), indicating no statistical difference between

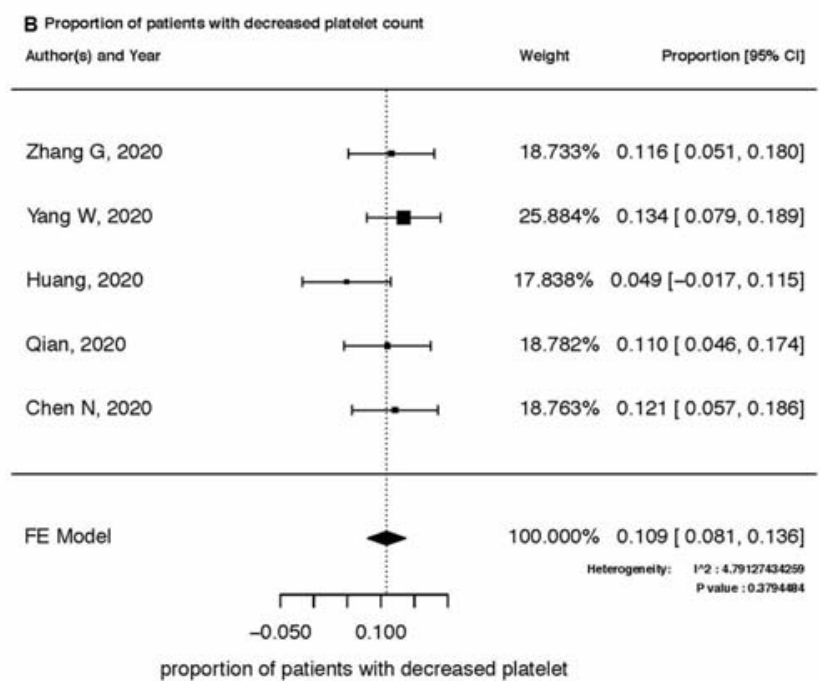

Fig. 3 (A) Forest plot of average platelet count of COVID-19 patients. (B) Forest plot of average proportion of COVID-19 patients with decreased platelet count. Heterogeneity is defined based on the $I^{2}$ index calculated. A random effect model is used to pool the average platelet count data, and a fixed effect model is used to pool the decreased platelet count database on the level of heterogeneity. 


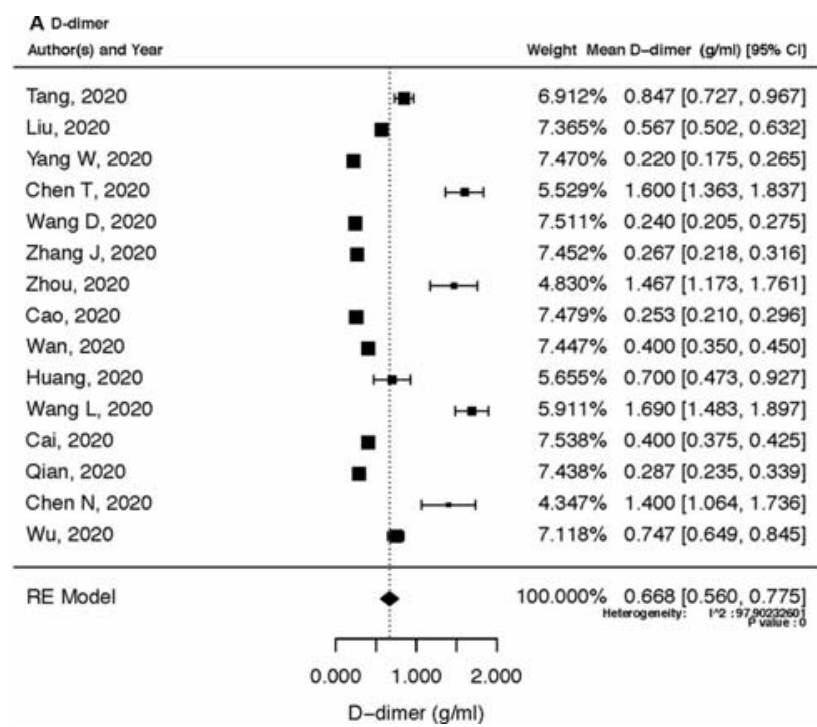

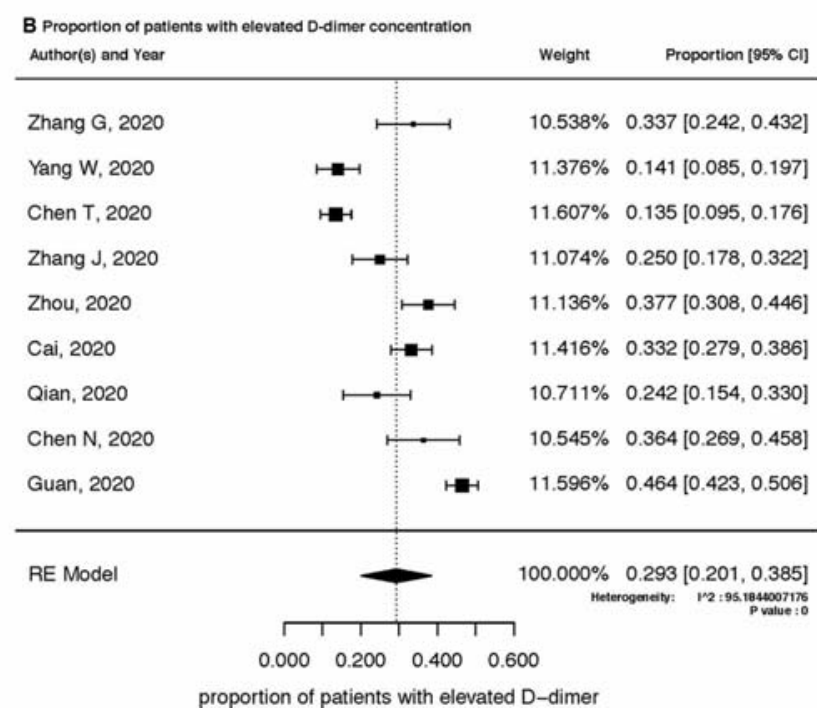

Fig. 4 (A) Forest plot of average D-dimer concentration of COVID-19 patients. (B) Forest plot of average proportion of COVID-19 patients with elevated D-dimer concentration. Heterogeneity is defined based on the $l^{2}$ index calculated, and random effect models are used to pool the database on the heterogeneity.

these two groups (-Fig. 5B). In addition, the D-dimer value in severe patients was $0.44 \mu \mathrm{g} / \mathrm{mL}(95 \% \mathrm{CI}: 0.23-0.66$, $Z=4.04, p<0.05)$, higher than that in nonsevere patients (-Fig. 5C). The PLT count in severe patients was $-14.47 \times 10^{9} / \mathrm{L}(95 \% \mathrm{CI}:-33.0-4.06, Z=-1.5, p=0.126)$, which was not significantly lower than that in nonsevere patients ( - Fig. 5D). For all comparisons above, the calculated $I^{2}$ indexes were between 68.42 and $86.91 \%$, indicating the presence of high heterogeneity within all the parameters we compared, thus random effect models were applied to pool the data.

Finally, we compared coagulation indicators and DIC incidence between survivors and nonsurvivors of COVID19 patients. The meta-analysis showed that nonsurvivors had an average PT of 1.23 seconds (95\% CI: $0.60-1.86$, $Z=3.84, p<0.05$ ), which is longer than that of survivors ( - Fig. 6A). The mean difference of APTT between nonsurvivors and survivors was 0.25 seconds $(95 \% \mathrm{CI}:-2.30-2.80$, $Z=0.19, p=0.85$ ), which indicates no significant difference of APTT between these two groups ( - Fig. 6B). Nonsurvivors also showed a D-dimer value of $5.91 \mu \mathrm{g} / \mathrm{mL}$ (95\% CI: 3.568.27), which was significantly higher than that of survivors (-Fig. 6C). A significant difference was also observed for PLT count between survivors and nonsurvivors. The mean difference of PLT count between survivors and nonsurvivors was $38.37 \times 10^{9} / \mathrm{L}(95 \% \mathrm{CI}:-55.79$ to $-20.94, Z=-4.3, p<0.05)$ ( - Fig. 6D). We also analyzed the incidence of DIC and the log risk ratio incidence of DIC in nonsurvivors compared with survivors. Based on the three studies that included data of DIC incidence, we found that $6.2 \%$ (95\% CI: 2.6-9.9\%) of the COVID-19 patients were complicated by DIC. The log risk ratio of DIC complicated in nonsurvivors compared with survivors was 3.267 (95\% CI: 2.19-4.34, $Z=5.95, p<0.05$ ), indicating that DIC is 26.2 times more likely to develop in nonsurvivors compared with survivors ( - Fig. 7 ). All the data used for the comparisons above showed calculated $I^{2}>50 \%$ except for the DIC risk ratio data. Therefore, the fixed effect model was applied to the DIC risk ratio data, whereas the random effect model was used to pool all the other data.

\section{Discussion}

Our meta-analysis showed that coagulation dysfunction in admitted Chinese COVID-19 patients is common, and the clinical manifestations are diverse, ranging from mild disorders of coagulation indicators to DIC.

\section{D-Dimer}

Our meta-analysis showed that the elevation of D-dimer was the most common coagulation abnormality. About one-third of COVID-19 patients had elevated D-dimer values on admission and the elevated D-dimer is closely associated with the severity and prognosis of COVID-19. Zhou and his colleagues have shown that D-dimer $>1.0 \mathrm{~g} / \mathrm{L}$ was an independent risk factor to identify patients with poor prognosis at the early stage. ${ }^{12}$ It was also found that the D-dimer was negatively correlated with 28-day mortality in multivariate analysis, ${ }^{13}$ and when the D-dimer value exceeds $3.0 \mu \mathrm{g} / \mathrm{mL}$ (sixfold of upper limit of normal [ULN]), heparin or low-molecular-weight heparin (LMWH) treatment can reduce mortality by approximately $20 \%$ (32.8 vs. $52.4 \%, p=0.017$ ). ${ }^{15}$ Therefore, a high D-dimer value above sixfold of ULN may be the appropriate timing for anticoagulation treatments of Chinese COVID-19 patients, which needs further prospective study to be confirmed.

\section{APTT and PT}

Different from SARS within which 50 to $63 \%$ of patients showed a prolonged level of APTT, our meta-analysis showed that the average PT and APTT in COVID-19 patients remained in the normal range. Our analysis also showed that severe patients and nonsurvivors had significantly longer PT than nonsevere patients and survivors, whereas APTT showed no significant difference. Chen et al have reported that 16 and 30\% COVID-19 patients exhibited shortened APTT and PT on 


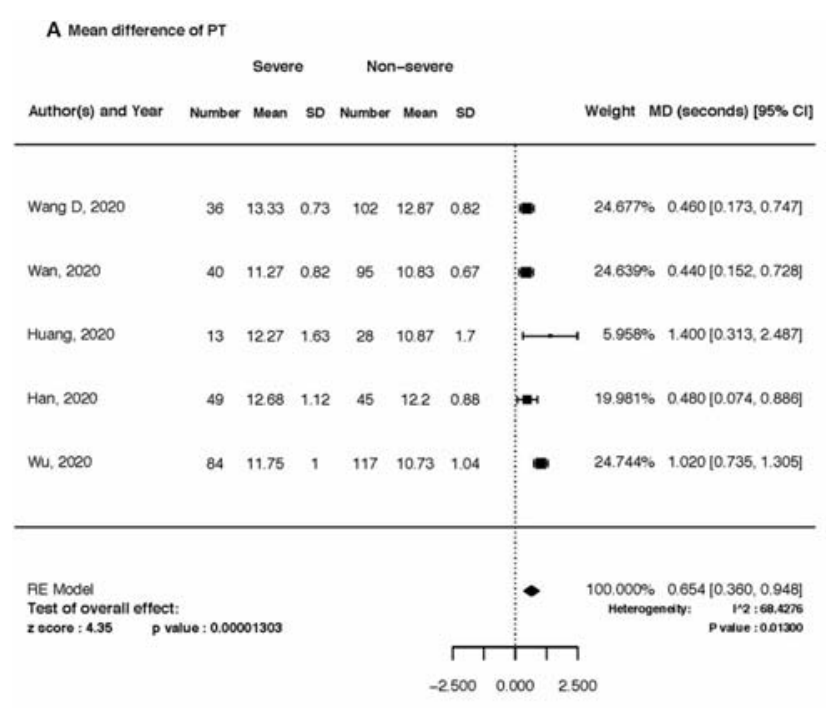

Non-severe patients with prodonged PT MD(seconds) Severe patients with proplonged PT

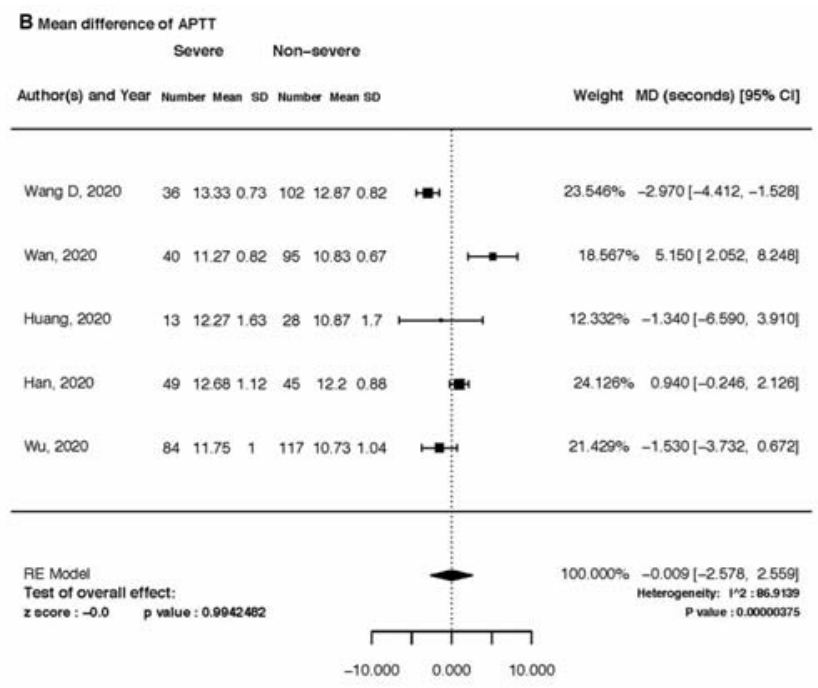

Non-severe patents with prolonged APTT MD(seconds) Severe patients with proplonged APTT

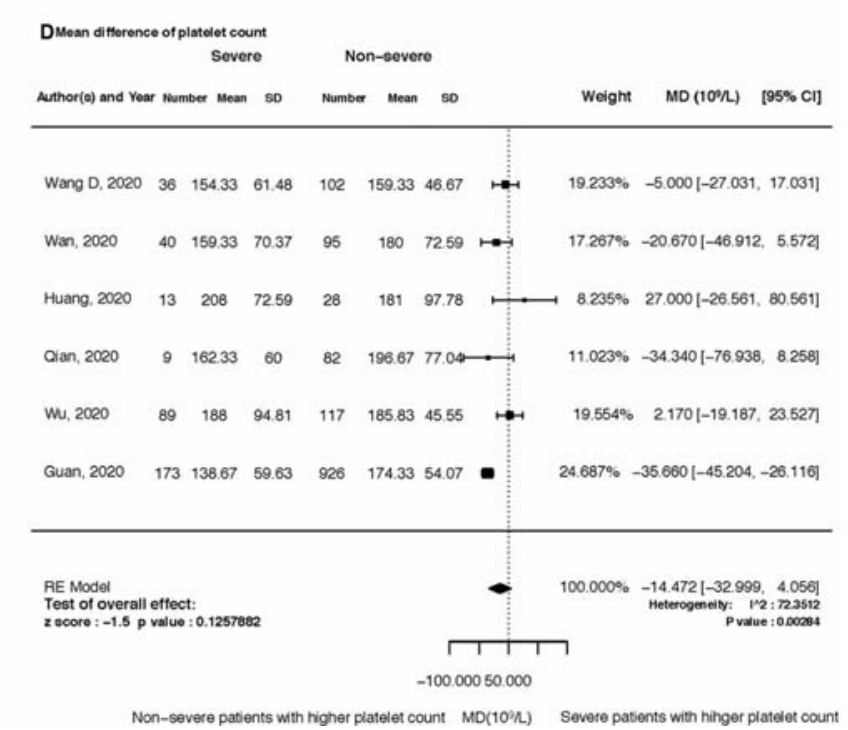

Non-severe patients with higher platelet count MD(10\%) Severe pasients with hinger platelet count

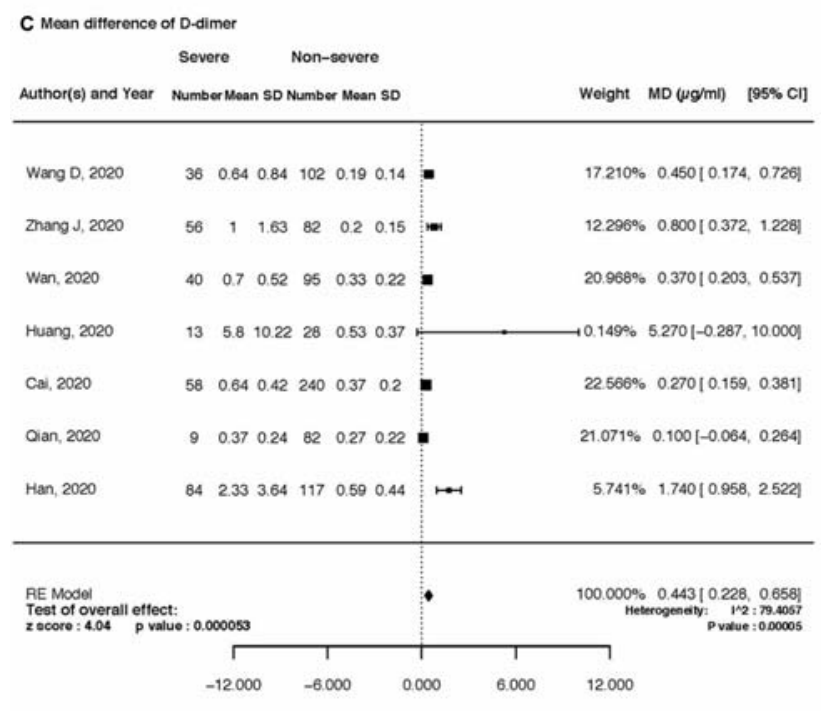

Non-severe patients with higher D-dimer value MD(ug/ml) Severe patients with higher D-dimer

Fig. 5 (A) Forest plot of mean difference of PT of severe patients compared with nonsevere patients. (B) Forest plot of mean difference of APTT of severe patients compared with nonsevere patients. (C) Forest plot of mean difference of D-dimer concentration of severe patients compared with nonsevere patients. (D) Forest plot of mean difference of platelet count of severe patients compared with nonsevere patients.

Heterogeneity is defined based on the $I^{2}$ index calculated, and random effect models are used to pool the database on the heterogeneity. APTT, activated partial thromboplastin time; PT, prothrombin time.

admission, respectively. ${ }^{20}$ The inconsistency of changes of PT and APTT may be attributed to the different sample populations and the different courses of the disease. COVID-19 patients in the early stage showed activation of the exogenous coagulation system, manifested as decreased PT and hypercoagulable state. Along with the progression of the disease, especially when patients develop DIC, PT significantly prolongs, which is associated with the poor prognosis of patients.

\section{Platelet}

It has been shown that the PLT count reached the level $<144 \times 10^{9} / \mathrm{L}$ in $45 \%$ of SARS patients at the time of onset and the decrease peaked after 1 week. ${ }^{10}$ Our meta-analysis showed that only $10.9 \%$ (95\% CI: $8.1-13.6 \%$ ) of COVID-19 patients had thrombocytopenia. Autopsy results indicated that the pathologic change of COVID-19 mainly concentrated in the lung where hyaline thrombus was observed within the blood vessels. This fibrinous thrombus is different from the white thrombus in the lungs of SARS patients, which might be one of the reasons that thrombocytopenia is less common in COVID-19 patients compared with SARS patients. Autopsy also showed that myelopoiesis was decreased in bone marrow, ${ }^{36}$ which is consistent with our meta-analysis results that the PLT count in nonsurvivors is significantly lower than that in survivors, indicating that thrombocytopenia is closely related to the mortality of COVID-19 patients. 
1532 Prevalence and Impact of Coagulation Dysfunction in COVID-19 jin et al.
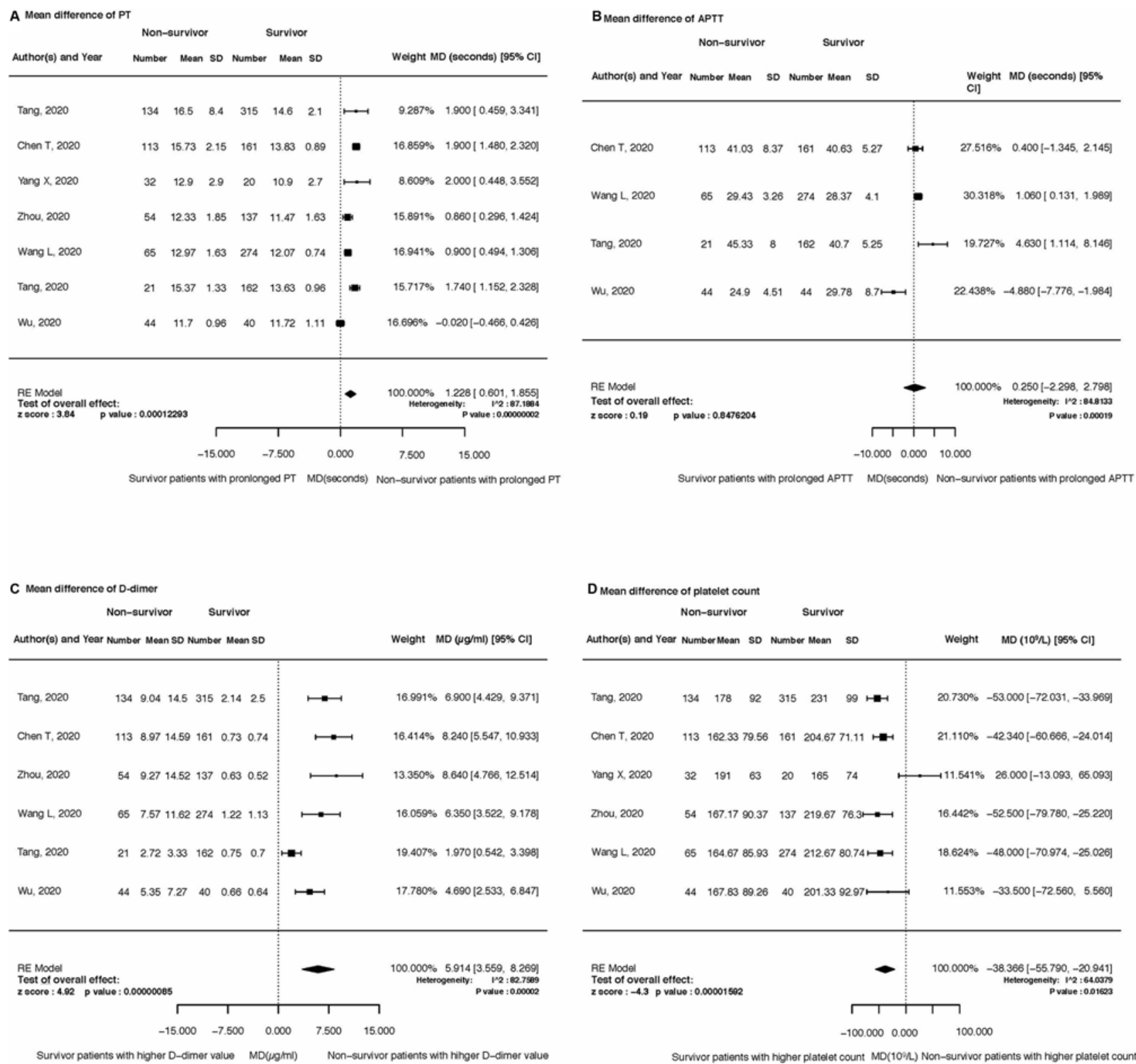

Fig. 6 (A) Forest plot of mean difference of PT of nonsurvivors compared with survivors. (B) Forest plot of mean difference of APTT of nonsurvivors compared with survivors. (C) Forest plot of mean difference of D-dimer concentration of nonsurvivors compared with survivors. (D) Forest plot of mean difference of platelet count of nonsurvivors compared with survivors. Heterogeneity is defined based on the $P^{2}$ index calculated, and random effect models are used to pool the database on the heterogeneity. APTT, activated partial thromboplastin time; PT, prothrombin time.

\section{Fibrinogen}

Our meta-analysis showed that the average fibrinogen concentration was in the normal range, which indicated that instead of hyperfibrinolysis seen in the late stage of DIC, fibrinolysis shutdown is the main feature along with the progression of COVID-19. The dysfunction of endothelial cells induced by infection results in excess thrombin generation and fibrinolysis shutdown, which lead to a hypercoagulable state in patients with infection. ${ }^{37}$

\section{DIC Occurrence}

Our meta-analysis showed that $6.2 \%$ of the COVID-19 patients were complicated by DIC, and the incidence was over 26 -fold higher in nonsurvivors than in survivors, which indicated that complication with DIC tends to be restricted to a late-stage disease. Therefore, monitoring specific coagulation and fibrinolysis biomarkers, such as soluble thrombomodulin, thrombinantithrombin complex, and plasminogen activator inhibitor-1, is necessary and helpful for the early diagnosis and a timely intervention of DIC. ${ }^{38}$

It has been reported that the incidence of deep venous thrombosis in SARS patients is approximately 20.5\%, and the incidence of pulmonary embolism is $11.4 \%{ }^{39}$ COVID-19 patients are also at high risk of venous thromboembolism (VTE) due to blood hypercoagulability conditions. Cui et al reported that the incidence of VTE in patients with severe COVID-19 admitted in the ICU was 25\% (20/81), and Ddimer $>1.5 \mu \mathrm{g} / \mathrm{mL}$ was a good indicator for identifying high- 

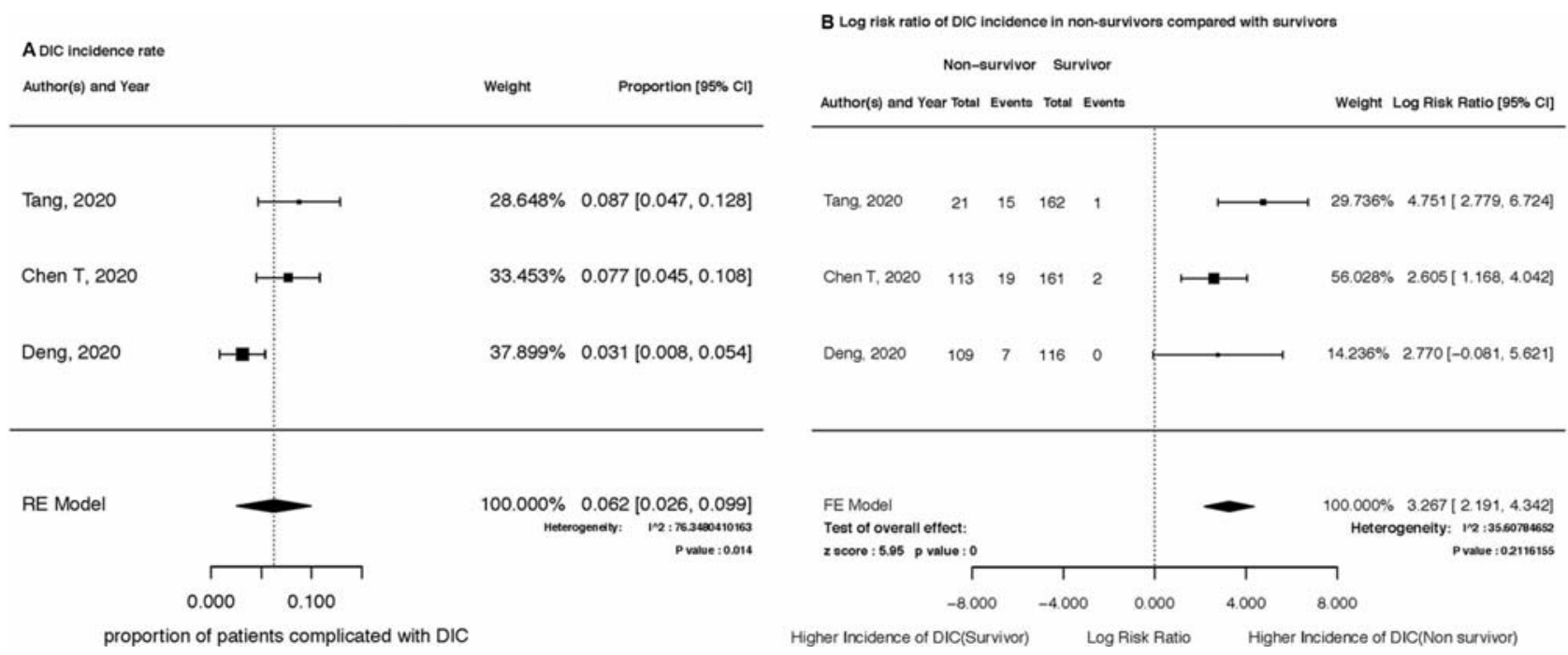

Fig. 7 (A) Forest plot of average DIC incidence of COVID-19 patients. (B) Forest plot of log risk ratio of DIC incidence in nonsurvivors compared with survivors. Heterogeneity is defined based on the $l^{2}$ index calculated. A random effect model is used to pool the average DIC incidence data, and a fixed effect model is used to pool the data that compares DIC incidence in nonsurvivors and survivors based on the level of heterogeneity. DIC, disseminated intravascular coagulation.

risk groups of VTE ${ }^{40}$ Klok et al also found that the cumulative incidence of the thrombotic complications in 184 COVID-19 patients admitted in the ICU was 31\% (95\% CI: $20-41 \%$ ), in which CTPA (computed tomography pulmonary angiography) and/or ultrasonography-confirmed VTE was $27 \%$ (95\% CI: $17-37 \%$ ). Age and coagulopathy were independent predictors of thrombotic complications. ${ }^{41}$ Thus, VTE cannot be ignored during the management of COVID-19, and VTE risk screening should be applied as early as possible. For all severe and critical COVID-19 cases and mild cases with estimation of high thrombus risk, pharmacological thrombosis prophylaxis should be applied if there are no contraindications. ${ }^{42}$

Based on the results of our meta-analysis and our frontline experience, we find that the manifestations of COVID-19-associated coagulopathy mainly include a hypercoagulant state, a tendency of thrombosis formation, and DIC induced by diffuse microvascular injury. Therefore, anticoagulation may be beneficial for the management of COVID-19 patients. Tang et al have reported that LMWH (mostly used in prophylactic rather than therapeutic doses) did not confer an overall survival advantage but was associated with improved survival in the patients with a sepsisinduced coagulopathy score $\geq 4$ and in those with D-dimer levels more than six times of the ULN. ${ }^{15}$ It was found that caucasian COVID-19 patients on prophylactic dose of LMWH treatment did not typically develop overt DIC. ${ }^{43}$ However, Klok et al suggested increasing the prophylaxis toward high-prophylactic doses for COVID-19 patients admitted to the ICU. ${ }^{41}$ Therefore, it is urgent to conduct adequately powered randomized controlled studies to determine the appropriate dose and timing of anticoagulation treatment for COVID-19-associated coagulopathy. At present, clinicians can follow the ISTH interim guidance for the recognition and management of coagulopathy in COVID-
$19{ }^{44} \mathrm{~A}$ more aggressive individualized strategy might be required in selected cases. ${ }^{45}$ Bleeding manifestations are not common despite coagulopathy for COVID-19 patients. However, Tang et al's study showed that at the late stages of COVID-19, 26.8\% (6/21) nonsurvivors had significant decreases in fibrinogen $(<1 \mathrm{~g} / \mathrm{L}) .{ }^{15}$ Therefore, thrombosis and bleeding may occur simultaneously during the late stages of overt DIC, and anticoagulation and risk of bleeding should be balanced in that case. If possible, thromboelastogram is helpful to guide anticoagulation treatment.

\section{Study Limitations}

The number of studies included was limited in terms of sample size, data availability, and methodologic quality, as all of the patients were from China. Given that thrombotic risk is significantly impacted by race, it will be better to include more studies with a broad geographic scope, to get a more comprehensive understanding of COVID-19-associated coagulopathy. In addition, all of the articles are retrospective studies, and more detailed patient information, particularly regarding the relationship of comorbidities with coagulopathy, was not available in most studies at the time of analyses.

\section{Conclusion}

Our meta-analysis identified the high prevalence of coagulopathy in Chinese COVID-19 inpatients, which is closely associated with the severity and prognosis. Therefore, it is necessary to increase awareness of coagulation dysfunction in COVID-19 patients. Routinely applying VTE risk screening, closely monitoring coagulation indicators for early recognition, and applying appropriate anticoagulation treatment may improve the prognosis of COVID-19 patients in China. 


\section{What Is known about this topic?}

- Previous literatures have shown that SARS patients were always complicated by coagulation disorders, which is associated with the prognosis.

- Coagulation dysfunction is also common in COVID-19 patients. But different studies have shown that changes of coagulation indicators in COVID-19 patients are inconsistent and the incidence of DIC ranges from 0.1 to $8.74 \%$.

\section{What does this paper add?}

- Our meta-analysis identified the high prevalence of coagulopathy in COVID-19 patients, which is closely associated with the severity and prognosis.

- It is necessary to increase awareness of coagulation dysfunction in COVID-19 patients.

- Routinely applying VTE risk screening, closely monitoring coagulation indicators for early recognition, and applying appropriate anticoagulation treatment may improve the prognosis of COVID-19 patients.

\section{Authors' Contributions}

All the authors have participated in literature retrieval and viewpoint discussion in this article. S.J. and Y.J. contributed in writing this article. Y.J. did the statistics. X.Y. revised the article. All authors read and approved the final manuscript.

\section{Note}

The study does not require ethical approval because the meta-analysis is based on published research and the original data are anonymous.

\section{Conflict of Interest}

None declared.

\section{Acknowledgment}

The authors would like to express their appreciation for all of the health care workers and other hospital staff as well as the local authorities for their efforts to combat the outbreak of COVID-19.

\section{References}

1 Zhu N, Zhang D, Wang W, et al; China Novel Coronavirus Investigating and Research Team. A novel coronavirus from patients with pneumonia in China, 2019. N Engl J Med 2020;382(08):727-733

2 Zhou P, Yang XL, Wang XG, et al. A pneumonia outbreak associated with a new coronavirus of probable bat origin. Nature 2020;579 (7798):270-273

3 WHO. WHO Director-General's remarks at the media briefing on 2019-nCoV on 11 February 2020. 2020. Available at: https://www. who.int/dg/speeches/detail/who-director-general-s-remarks-at-themedia-briefing-on-2019-ncov-on-11-february-2020. Accessed February 11,2020
4 WHO. Coronavirus disease (COVID-19): Situation Report - 104. 2020. Available at: https://www.who.int/docs/default-source/ coronaviruse/situation-reports/20200503-covid-19-sitrep-104. pdf?sfvrsn=53328f46_2. Accessed March 30, 2020

5 Weston S, Frieman MB. COVID-19: knowns, unknowns, and questions. MSphere 2020;5(02):e00203-20

6 Jiang F, Deng L, Zhang L, Cai Y, Cheung CW, Xia Z. Review of the clinical characteristics of Coronavirus Disease 2019 (COVID-19). J Gen Intern Med 2020;35(05):1545-1549

7 Petrosillo N, Viceconte G, Ergonul O, Ippolito G, Petersen E. COVID-19, SARS and MERS: are they closely related? Clin Microbiol Infect 2020;26(06):729-734

8 WHO. Summary of probable SARS cases with onset of illness from 1 November 2002 to 31 July 2003. 2020. Available at: https:// www.who.int/csr/sars/country/table2004_04_21/en/. Accessed January 27, 2020

9 Lee N, Hui D, Wu A, et al. A major outbreak of severe acute respiratory syndrome in Hong Kong. N Engl J Med 2003;348(20): 1986-1994

10 Wong RS, Wu A, To KF, et al. Haematological manifestations in patients with severe acute respiratory syndrome: retrospective analysis. BMJ 2003;326(7403):1358-1362

11 Wang D, Hu B, Hu C, et al. Clinical characteristics of 138 hospitalized patients with 2019 novel coronavirus-infected pneumonia in Wuhan, China. JAMA 2020;323(11):1061-1069

12 Zhou F, Yu T, Du R, et al. Clinical course and risk factors for mortality of adult inpatients with COVID-19 in Wuhan, China: a retrospective cohort study. Lancet 2020;395(10229):1054-1062

13 Tang N, Li D, Wang X, Sun Z. Abnormal coagulation parameters are associated with poor prognosis in patients with novel coronavirus pneumonia. J Thromb Haemost 2020;18(04):844-847

14 Guan WJ, Ni ZY, Hu Y, et al; China Medical Treatment Expert Group for Covid-19. Clinical characteristics of Coronavirus Disease 2019 in China. N Engl J Med 2020;382(18):1708-1720

15 Tang N, Bai H, Chen X, Gong J, Li D, Sun Z. Anticoagulant treatment is associated with decreased mortality in severe Coronavirus Disease 2019 patients with coagulopathy. J Thromb Haemost 2020;18(05):1094-1099

16 Zhang G, Zhang J, Wang B, Zhu X, Wang Q, Qiu S. Analysis of clinical characteristics and laboratory findings of 95 cases of 2019 novel coronavirus pneumonia in Wuhan, China: a retrospective analysis. Respir Res 2020;21(01):74

17 Liu W, Tao ZW, Wang L, et al. Analysis of factors associated with disease outcomes in hospitalized patients with 2019 novel coronavirus disease. Chin Med J (Engl) 2020;133(09):1032-1038

18 Shi S, Qin M, Shen B, et al. Association of cardiac injury with mortality in hospitalized patients with COVID-19 in Wuhan, China. JAMA Cardiol 2020. Doi: 10.1001/jamacardio.2020.0950

19 Yang W, Cao Q Qin L, et al. Clinical characteristics and imaging manifestations of the 2019 novel coronavirus disease (COVID19): a multi-center study in Wenzhou city, Zhejiang, China. J Infect 2020;80(04):388-393

20 Chen T, Wu D, Chen H, et al. Clinical characteristics of 113 deceased patients with Coronavirus Disease 2019: retrospective study. BMJ 2020;368:m1091

21 Zhang JJ, Dong X, Cao YY, et al. Clinical characteristics of 140 patients infected with SARS-CoV-2 in Wuhan, China. Allergy 2020. Doi: $10.1111 /$ all.14238

22 Deng Y, Liu W, Liu K, et al. Clinical characteristics of fatal and recovered cases of Coronavirus Disease 2019 in Wuhan, China: a retrospective study. Chin Med J (Engl) 2020;133(11):1261-1267

23 Yang X, Yu Y, Xu J, et al. Clinical course and outcomes of critically ill patients with SARS-CoV-2 pneumonia in Wuhan, China: a single-centered, retrospective, observational study. Lancet Respir Med 2020;8(05):475-481

24 Cao J, Tu WJ, Cheng W, et al. Clinical features and short-term outcomes of 102 patients with corona virus disease 2019 in Wuhan, China. Clin Infect Dis 2020. Doi: 10.1093/cid/ciaa243 
25 Wan S, Xiang Y, Fang W, et al. Clinical features and treatment of COVID-19 patients in northeast Chongqing. J Med Virol 2020;92 (07):797-806

26 Huang C, Wang Y, Li X, et al. Clinical features of patients infected with 2019 novel coronavirus in Wuhan, China. Lancet 2020;395 (10223):497-506

27 Wang L, He W, Yu X, et al. Coronavirus Disease 2019 in elderly patients: characteristics and prognostic factors based on 4-week follow-up. J Infect 2020;80(06):639-645

28 Cai Q Huang D, Ou P, et al. COVID-19 in a designated infectious diseases hospital outside Hubei Province, China. Allergy 2020. Doi: 10.1111/all.14309

29 Qian GQ Yang NB, Ding F, et al. Epidemiologic and clinical characteristics of 91 hospitalized patients with COVID-19 in Zhejiang, China: a retrospective, multi-centre case series. QJM 2020. Doi: $10.1093 /$ qjmed/hcaa089

30 Chen N, Zhou M, Dong X, et al. Epidemiological and clinical characteristics of 99 cases of 2019 novel coronavirus pneumonia in Wuhan, China: a descriptive study. Lancet 2020;395 (10223):507-513

31 Han H, Yang L, Liu R, et al. Prominent changes in blood coagulation of patients with SARS-CoV-2 infection. Clin Chem Lab Med 2020; 58(07):1116-1120

32 Wu C, Chen X, Cai Y, et al. Risk factors associated with acute respiratory distress syndrome and death in patients with Coronavirus Disease 2019 pneumonia in Wuhan, China. JAMA Intern Med 2020. Doi: 10.1001/jamainternmed.2020.0994

33 Wan X, Wang W, Liu J, Tong T. Estimating the sample mean and standard deviation from the sample size, median, range and/or interquartile range. BMC Med Res Methodol 2014;14:135

34 Zhang Z, Wu P, Zhang J, Wang S, Zhang G. The effect of statins on microalbuminuria, proteinuria, progression of kidney function, and all-cause mortality in patients with non-end stage chronic kidney disease: a meta-analysis. Pharmacol Res 2016;105:74-83

35 Higgins JP, Thompson SG, Deeks JJ, Altman DG. Measuring inconsistency in meta-analyses. BMJ 2003;327(7414):557-560
36 Wang HJ, Du SH, Yue X, Chen CX. Review and prospect of pathological features of corona virus disease. Fa Yi Xue Za Zhi 2020;36(01):16-20

37 Schmitt FCF, Manolov V, Morgenstern J, et al. Acute fibrinolysis shutdown occurs early in septic shock and is associated with increased morbidity and mortality: results of an observational pilot study. Ann Intensive Care 2019;9(01):19

38 Iba T, Di Nisio M, Thachil J, et al. A proposal of the modification of Japanese Society on Thrombosis and Hemostasis (JSTH) disseminated intravascular coagulation (DIC) diagnostic criteria for sepsis-associated DIC. Clin Appl Thromb Hemost 2018;24(03): 439-445

39 Zhang Y, Xiao M, Zhang S, et al. Coagulopathy and antiphospholipid antibodies in patients with Covid-19. N Engl J Med 2020;382 (17): e38

40 Cui S, Chen S, Li X, Liu S, Wang F. Prevalence of venous thromboembolism in patients with severe novel coronavirus pneumonia. J Thromb Haemost 2020;18(06):1421-1424

41 Klok FA, Kruip MJHA, van der Meer NJM, et al. Incidence of thrombotic complications in critically ill ICU patients with COVID-19. Thromb Res 2020;191:145-147

42 Zhai Z, Li C, Chen Y, et al; Prevention Treatment of VTE Associated with COVID-19 Infection Consensus Statement Group. Prevention and treatment of venous thromboembolism associated with Coronavirus Disease 2019 infection: a consensus statement before guidelines. Thromb Haemost 2020;120(06):937-948

43 Fogarty H, Townsend L, Ni Cheallaigh C, et al. More on COVID-19 coagulopathy in Caucasian patients. Br J Haematol 2020;189(06): 1060-1061

44 Thachil J, Tang N, Gando S, et al. ISTH interim guidance on recognition and management of coagulopathy in COVID-19. J Thromb Haemost 2020;18(05):1023-1026

45 Kollias A, Kyriakoulis KG, Dimakakos E, Poulakou G, Stergiou GS, Syrigos K. Thromboembolic risk and anticoagulant therapy in COVID-19 patients: emerging evidence and call for action. Br J Haematol 2020;189(05):846-847 